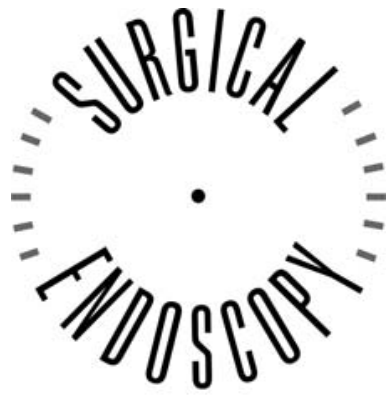

and Other Interventional Techniques

\title{
A prospective randomized comparison of two instruments for dissection and vessel sealing in laparoscopic colorectal surgery
}

\author{
M. Hübner, D. Hahnloser, F. Hetzer, M. K. Müller, P.-A. Clavien, N. Demartines \\ Department of Visceral and Transplantation Surgery, University Hospital of Zürich, Rämistrasse 100, 8091 Zürich, Switzerland
}

Received: 31 March 2006/Accepted: 27 April 2006/Online publication: 6 February 2007

\begin{abstract}
Background: A newly available, laparoscopic 5-mm bipolar vessel sealing device promises substantial advantages over the $10-\mathrm{mm}$ instrument. This study compared the safety as well as the technical and surgical aspects of these different tools.

Methods: For this study, 30 consecutive patients undergoing laparoscopic left-sided colectomy were prospectively randomized for the 5-mm LigaSure or The 10-mm LigaSure. The patients' demographics were analyzed together with their intraoperative and postoperative parameters, and the instruments were assessed by the surgeons with a standardized questionnaire.

Results: The two groups were comparable and demonstrated similar mean operation times, blood losses, and hospital stays. The 5-mm LigaSure was applied in more operation steps and resulted in fewer bleeding episodes and less lens cleaning. Monopolar scissors were used less frequently in the $5-\mathrm{mm}$ group, thus minimizing cauteric lesions and their complications ( 0 in the $5-\mathrm{mm}$ group vs 2 in the $10-\mathrm{mm}$ group). Overall satisfaction with the 5 -mm LigaSure was significantly higher $(8.4 \pm 0.18$ vs $6.9 \pm 0.41$ out of $10 ; p=0.002$ ), with significant advantages in terms of dissection capacity, visibility, and handling.

Conclusion: The 5-mm LigaSure is as secure and fast as the larger $10-\mathrm{mm}$ device and compares favorably in terms of finer dissection as well as trocar flexibility and handling. Therefore, it can be used safely in laparoscopic colorectal surgery.
\end{abstract}

Key words: Colorectal — Laparoscopy — LigaSure Sigmoidectomy — Vessel sealing
Bipolar vessel sealing (LigaSure; Valleylab, Boulder, CO, USA) has gained wide acceptance as an alternative technique for hemostasis and fast preparation in open and laparoscopic surgery $[2,4,6-9,11]$. The main advantages are limited collateral tissue damage and secure, visible, and undisplaceable sealing with bursting strengths superior to ultrasonic and bipolar vessel occlusions and comparable with those of ligatures and clips $[3,5,7]$.

For laparoscopic procedures, until recently, only a device with a shaft diameter of $10 \mathrm{~mm}$ was available. The major drawbacks of the $10-\mathrm{mm}$ instrument are its shape (straight jaw angle); its size, which hampers a meticulous dissection; the restriction to $10-\mathrm{mm}$ trocars exclusively; and the lack of a finger trigger, making the use of a food pedal necessary. The new tool with a shaft diameter of $5 \mathrm{~mm}$ has a curved jaw angle and a finger trigger. The shaft is longer ( 37 vs $32 \mathrm{~cm}$ ), but the electrode is smaller (18 vs $22 \mathrm{~mm}$ ), as is the seal width (2-4 vs $6 \mathrm{~mm}$ ). According to the producer, a secure seal of tissue bundles and vessels up to $7 \mathrm{~mm}$ with a collateral damage of $2 \mathrm{~mm}$ is equally warranted for both instruments.

This prospective randomized study aimed to assess the new 5-mm LigaSure in laparoscopic colorectal surgery, and to compare it with the $10-\mathrm{mm}$ instrument in terms of safety, intraoperative complications, and comfort of the surgeon.

\section{Materials and methods}

For this study, 30 consecutive patients undergoing elective laparoscopic left-sided colectomy (60\% for recurrent diverticulitis, $40 \%$ for sigmoid cancer) between September 2004 and May 2005 were randomized by closed envelope to 5-mm LigaSure or 10-mm LigaSure, respectively. The authors did not receive any financial support from the manufacturer. The study was approved by the institutional ethical board.

Pre-, intra-, and postoperative parameters were assessed prospectively for all the patients. All the procedures were performed by three expert laparoscopic surgeons in a standardized manner using a four-trocar technique. The surgeon documented the use of LigaSure, monopolar scissors, or sutures/clips for each step of the operation 
(vessel dissection, distal and proximal mobilization of the colon/rectum). Similarly, lens cleaning, the need of additional trocars, and the occurrence of bleeding episodes were recorded. The surgeon graded the instrument used on a visual analog scale (VAS) from 0 (poor) to 10 (outstanding) with regard to dissection capacity, sealing, cutting, visibility conditions, speed of dissection, management of bleeding, handgrip, seal and cut width, handling, limited space dissection, security, reliability, overall satisfaction, and main advantages/disadvantages of the instrument used.

Statistical analysis was performed using standard software SPSS 8.0 (SPSS, Inc., Chicago, IL) for Windows. To compare continuous variables between the two groups, the Mann-Whitney $U$ test was used. Categorical variables were compared using the chi-square test or, when appropriate, Fisher's exact test. Results are expressed as median and range unless otherwise stated. A $p$ value less than 0.05 was considered to indicate statistical significance.

\section{Results}

\section{Patient and operation demographics}

Both groups were similar with regard to age, body mass index (BMI), gender distribution, American Society of Anesthesiology (ASA) score, indication for surgery, and type of operation (rectosigmoid or low anterior resection). In addition, preoperative prothrombin time and platelets were not significantly different between the two groups.

The two groups were comparable in terms of operation time (median, $200 \mathrm{~min}$; range, 110-330 min for the 5-mm group vs median, $210 \mathrm{~min}$; range, 160-420 min for the 10-mm group, $p=0.68$ ), estimated blood loss (median, $100 \mathrm{ml}$; range, 25-300 $\mathrm{ml}$ vs median, $200 \mathrm{ml}$; range, $50-400 \mathrm{ml} ; p=0.09$ ), delta hematocrit (median, $6 \%$; range, $0-11 \%$ vs median, $7 \%$; range, $0-10 \%$, preoperative-postoperative; $p=0.8$ ), and length of hospital stay (median, 5 days; range, 3-14 days vs median, 6 days; range, 3-28 days; $p=0.23$ ).

\section{Complications}

There were two intraoperative complications in the 5-mm group. One serosal laceration caused by the grasper had to be sutured, and one resection had to be extended because of ischemia. In the 10-mm group, one bladder perforation was oversewn, and one conversion to open surgery was necessary to guarantee an oncologic resection. One patient in the 5-mm group underwent laparoscopic reoperation for a small bowel ileus (complication grade $3 \mathrm{~b}$ according to Dindo et al. [1]), and one patient in the $10-\mathrm{mm}$ group had a laparotomy for a small bowel perforation attributable to a cauteric lesion during take-down of adhesions (grade $3 \mathrm{~b}$ ). In addition, three patients had a wound infection $(5 \mathrm{~mm}$, one patient; $10 \mathrm{~mm}$, two patients; grade 2), and one patient had a lower leg compartment syndrome and underwent an emergency fasciotomy (grade $3 b$ ).

\section{Operative parameters}

The objective intraoperative events are displayed in Fig. 1. Bleeding episodes, use of sutures/clips, and the

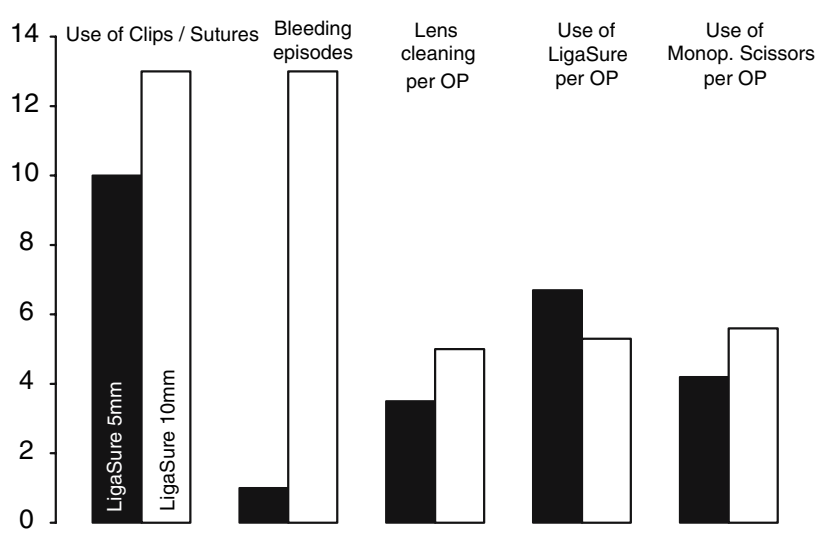

Fig. 1. Intraoperative events. The number of the following events (use of/occurrence of) is displayed for the 5-mm LigaSure (black) versus the 10-mm device (white): Application of sutures/clips and bleeding episodes are for the total group of 15 patients each. The bars for lens cleaning and use of LigaSure and monopolar scissors, respectively, state the mean value per operation.

necessity of lens cleaning were less frequent in the 5-mm group, but the difference was not statistically significant. Overall, the 5-mm LigaSure was applied in more steps of the operation than the $10-\mathrm{mm}$ instrument, whereas the monopolar scissors had to be used more frequently in the $10-\mathrm{mm}$ group. Additional trocars were placed in two patients of the 5-mm group and three patients of the 10-mm group.

\section{Surgeons' evaluation}

Overall satisfaction for both instruments was $7.6 \pm 1.4$ out of a maximum of 10, with the best estimation for the sealing capacity $(8.6 \pm 2.2)$ and the worst value for handling of the instrument $(7.0 \pm 2.2)$. The $5-\mathrm{mm}$ LigaSure was significantly favored by the surgeons with regard to the criteria dissection $(8.7 \pm 0.24$ vs $6.2 \pm 0.67 ; \quad p=0.004)$, visibility conditions $(8.4 \pm 0.14$ vs $7.3 \pm 0.41 ; \quad p=0.019)$, handgrip $(7.9 \pm 0.52$ vs $6.3 \pm 0.35 ; \quad p=0.005)$, handling $(8.5 \pm 0.40$ vs $5.7 \pm 0.54 ; p=0.001)$, limited space for dissection $(8.2 \pm 0.22$ vs $6.1 \pm 0.61 ; p=0.001)$, and overall satisfaction $(8.4 \pm 0.18$ vs $6.9 \pm 0.41 ; p=$ $0.002)$.

There was no statistically significant difference in sealing and cutting capacity, speed of dissection, management of bleeding, seal and cut width, security, or reliability. The subjective evaluation of the two instruments is illustrated in Fig. 2 as the difference of the mean values.

The surgeons listed as main advantages of the 5-mm instrument its trocar flexibility in 10 of 15 operations and ease of dissection (6/15), whereas they complained about its slow speed (6/15) and short seal/cut width (5/ $15)$. On the other hand, the strengths of the $10-\mathrm{mm}$ LigaSure were its security (7/15), sealing capacity $(6 / 15)$, and speed of dissection (5/15), especially in overweight patients, whereas the clumsy dissection (8/15) and lack of trocar flexibility $(7 / 15)$ were regarded as serious drawbacks of the instrument. 


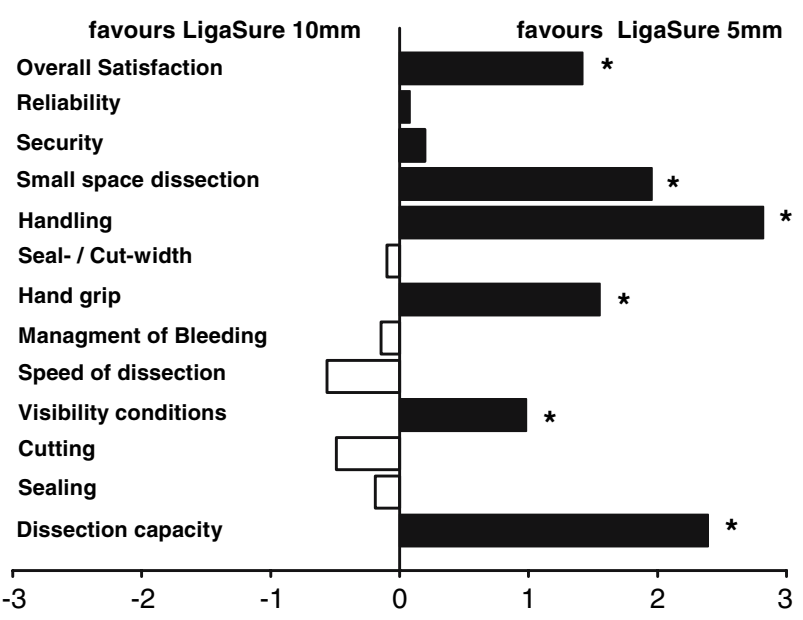

Fig. 2. Subjective evaluation the $5-\mathrm{mm}$ LigaSure versus the $10-\mathrm{mm}$ device. The evaluated criteria are given in the legend and displayed as differences in the mean values. The negative values (white boxes going to the left) indicate the advantages of the 10-mm LigaSure, whereas the positive values (black boxes going to the right) are in favor of the 5 -mm LigaSure. Statistically significant differences $(p<0.05)$ are marked $(*)$.

\section{Discussion}

This is the first prospective randomized comparison of two instruments for dissection and vessel sealing in laparoscopic colorectal surgery. Takada et al. [12] compared the $10-\mathrm{mm}$ LigaSure and ultrasonic coagulation shears $(10 \mathrm{~mm})$ for laparoscopic colectomy and found a reduced dissection time with the LigaSure. However, the patients were not randomized, and both the total operation time and evaluation of the instruments were unfortunately not stated. Morino et al. [10] reported less intraoperative blood loss in laparoscopic colorectal operations with ultrasonic dissection than with standard electrosurgery. The operation time, however, did not differ significantly between the two groups. In our study, the overall operation time and blood loss were similar in both groups and comparable with the literature $[10,13]$. Despite different seal width and electrode length, no differences in terms of operation time, speed of dissection, or control of bleeding were observed. Bleeding episodes occurred less frequently with the smaller instrument, probably due to the possibility of a finer dissection. This also is supported by significantly higher subjective scores for dissection, especially in a small surgical space such as the pelvis.

In addition, the 5-mm device was used during more operation steps than the larger $10-\mathrm{mm}$ instrument, making changes of instruments less frequent. The 5-mm device could be used through all trocars, thus allowing the use of at least two 5-mm trocars (lower cost and smaller scar). Moreover, the longer shaft eased the mobilization of the splenic flexure. For all these reasons, monopolar scissors had to be used more frequently in the $10-\mathrm{mm}$ group, which may explain two electrocautery complications (one bladder perforation and one small bowel perforation), whereas no cauteric lesion was observed in the $5-\mathrm{mm}$ group. The better visibility conditions with the $5-\mathrm{mm}$ device probably are attributable to the smaller and curved forceps and less smoke production. Not surprisingly, the size of the instrument had no influence on the length of hospital stay or on direct or related costs (both instruments are priced the same).

Certainly, the surgeons' individual preferences for the two instruments may entail a bias. However, the groups were well randomized, and only three different surgeons performed the operations. Another criticism might be the small number of patients and the lack of a power analysis, but no preliminary data were available. To our knowledge, this is the only randomized study taking into account objective pre- and postoperative parameters, outcome, and surgeons' preferences.

We conclude that the new 5-mm LigaSure is as secure and fast as the larger 10-mm device and compares favorably with regard to finer dissection, trocar flexibility, and handling. Therefore, it can be safely used in laparoscopic colorectal surgery.

\section{References}

1. Dindo D, Demartines N, Clavien PA (2004) Classification of surgical complications: a new proposal with evaluation in a cohort of 6,336 patients and results of a survey. Ann Surg 240: 205-213

2. Fleshman J (2002) Advanced technology in the management of hemorrhoids: stapling, laser, harmonic scalpel, and LigaSure. J Gastrointest Surg 6: 299-301

3. Harold KL, Pollinger H, Matthews BD, Kercher KW, Sing RF, Heniford BT (2003) Comparison of ultrasonic energy, bipolar thermal energy, and vascular clips for the hemostasis of small-, medium-, and large-sized arteries. Surg Endosc 17: 1228-1230

4. Heniford BT, Matthews BD, Sing RF, Backus C, Pratt B, Greene FL (2001) Initial results with an electrothermal bipolar vessel sealer. Surg Endosc 15: 799-801

5. Kennedy JS, Stranahan PL, Taylor KD, Chandler JG (1998) High-burst-strength, feedback-controlled bipolar vessel sealing. Surg Endosc 12: 876-878

6. Kirdak T, Korun N, Ozguc H (2005) Use of LigaSure in thyroidectomy procedures: results of a prospective comparative study. World J Surg 29(6): 771-774

7. Landman J, Kerbl K, Rehman J, Andreoni C, Humphrey PA, Collyer W, Olweny E, Sundaram C, Clayman RV (2003) Evaluation of a vessel sealing system, bipolar electrosurgery, harmonic scalpel, titanium clips, endoscopic gastrointestinal anastomosis vascular staples and sutures for arterial and venous ligation in a porcine model. J Urol 169: 697-700

8. Lee WJ, Chen TC, Lai IR, Wang W, Huang MT (2003) Randomized clinical trial of LigaSure versus conventional surgery for extended gastric cancer resection. Br J Surg 90: 1493-1496

9. Matthews BD, Pratt BL, Backus CL, Kercher KW, Mostafa G, Lentzner A, Lipford EH, Sing RF, Heniford BT (2001) Effectiveness of the ultrasonic coagulating shears, LigaSure vessel sealer, and surgical clip application in biliary surgery: a comparative analysis. Am Surg 67: 901-906

10. Morino M, Rimonda R, Allaix ME, Giraudo G, Garrone C (2005) Ultrasonic versus standard electric dissection in laparoscopic colorectal surgery: a prospective randomized clinical trial. Ann Surg 242: 897-901; discussion 901

11. Romano F, Franciosi C, Caprotti R, Uggeri F (2005) Hepatic surgery using the Ligasure vessel sealing system. World J Surg 29: $110-112$

12. Takada M, Ichihara T, Kuroda Y (2005) Comparative study of electrothermal bipolar vessel sealer and ultrasonic coagulating shears in laparoscopic colectomy. Surg Endosc 19: 226-228

13. Tekkis PP, Senagore AJ, Delaney CP, Fazio VW (2005) Evaluation of the learning curve in laparoscopic colorectal surgery: comparison of right-sided and left-sided resections. Ann Surg 242: 83-91 